\title{
Resiliencia comunitaria: defensa del agua y del territorio en la cuenca del río Sumapaz, Colombia
}

\author{
COMMUNITY RESILIENCE: WATER AND TERRITORY DEFENSE IN THE \\ SUMAPAZ RIVER BASIN, COLOMBIA
}

\author{
RESILIÊNCIA COMUNITÁRIA: DEFESA DA ÁGUA E DO TERRITÓRIO NA \\ BACIA DO RIO SUMAPAZ, COLÔMBIA
}

Para citar este artículo: Botia Flechas, C. J. y Preciado Beltran, J. (2019).

Clara Judyth Botia Flechas ${ }^{1}$

Jair Preciado Beltrán ${ }^{2}$

Resiliencia comunitaria: defensa del agua y del territorio en la cuenca

del río Sumapaz, Colombia. Perspectiva Geográfica, 24(1), 13-34.

https://doi.org/10.19053/01233769.8425.

Recepción:

26 de enero de 2018

Evaluación:

19 de septiembre de 2018

Aprobación:

30 de noviembre de 2018

\section{Resumen}

El objetivo central de este artículo es analizar la resiliencia comunitaria de los actores locales y comunitarios de la cuenca del río Sumapaz a partir de los procesos de defensa del agua y del territorio ocurridos en el periodo comprendido entre los años 2012-2017 por la implantación los proyectos mineros, de exploración de hidrocarburos y de generación de energía hidroeléctrica. La metodología se basó en la revisión documental del contexto histórico y los eventos recientes, a nivel nacional y local, relacionados con el tema, así como la aplicación de entrevistas semiestructuradas a actores clave. Para el análisis de la resiliencia comunitaria se escogieron las categorías: identidad cultural, autoestima colectiva, cohesión social y prácticas de

1 Doctora en Geografía de la Universidad Pedagógica y Tecnológica de Colombia en convenio con el Instituto Geográfico Agustín Codazzi. Docente de la Universidad Distrital Francisco José de Caldas.cjbotiaf@udistrital.edu.co.

2 Doctor en Geografía de la Universidad Estadual Paulista. Docente de la Universidad Distrital Francisco José de Caldas. jair.preciado@gmail.com 
democracia participativa. La investigación evidenció que en la cuenca del río Sumapaz la resiliencia comunitaria se traduce en un fortalecimiento de los actores locales y comunitarios que comparten preocupaciones en torno al agua y se apropian de sus realidades territoriales para construir una conciencia crítica sobre el ser, el estar, el hacer y el transformar en su territorio.

Palabras clave: agua, cuenca del río Sumapaz, resiliencia comunitaria, territorio.

\section{Abstract}

The aim of this article is to analyze community resilience of local and community actors in the Sumapaz river basin from water and territory defense processes that took place between 2012-2017 due to the implementation of mining, oil and gas exploration, and hydroelectric power generation projects. The method employed involves a documentary review of the historical context and recent events nationally and locally, as well as semi-structured interviews with key actors. To examine community resilience, categories chosen include cultural identity, collective self-esteem, social cohesion, and participatory democracy practices. Research shows that, in the Sumapaz river basin, community resilience translates into the strengthening of local and community actors who share their water concerns and take hold of their territorial realities in order to raise awareness of their being, doing and transforming the territory.

Keywords: Water, Sumapaz river basin, community resilience, territory.

\section{Resumo}

O objetivo principal do presente artigo é analisar a resiliência da comunidade dos atores locais e comunitários na bacia do rio Sumapaz a partir dos processos de defesa da água e do território ocorridos no período compreendido entre os anos 2012-2017 pela implantação dos projetos de mineração, de exploração de hidrocarbonetos e de geração de energia hidrelétrica. A metodologia baseou-se na revisão documental do contexto histórico e dos eventos recentes, em nível nacional e local, relacionados ao assunto, bem como na aplicação de entrevistas semiestruturadas aos atores chave. Para a análise da resiliência comunitária, escolheram-se as categorias: identidade cultural, autoestima coletiva, coesão social e práticas de democracia participativa. A pesquisa evidenciou que na bacia do rio Sumapaz, a resiliência da comunidade 
se traduz em um fortalecimento dos atores locais e comunitários que compartilham preocupações referentes à água e apropriam-se de suas realidades territoriais para criar uma consciência crítica sobre o ser, o ficar, o fazer e o transformar em seu território.

Palavras chave: Água, bacia do rio Sumapaz, resiliência comunitária, território.

\section{Introducción}

Desde comienzos de la década 1970 hasta hoy, la discusión sobre la crisis ambiental global ha estado asociada a los procesos económicos, sociales y políticos y sus complejas interacciones. La globalización ha transformado el ambiente y ha generado luchas sociales por la propiedad y el control de los recursos naturales, lo que ha obligado a las comunidades a desarrollar estrategias de autogestión de su patrimonio territorial local (Leff, 2004). La globalización ha generado la unidad de mundo, universalizando los lugares y fundiendo el tiempo y el espacio (Santos, 1995). En este contexto, la problemática espacial surge como una crisis del capitalismo que se presenta en el momento en que las relaciones de producción ya no se pueden reproducir ampliamente y la lucha de clases se centra, de acuerdo con Soja y Hadjimichalis (1979), en la producción del espacio, el derecho a la ciudad, la estructura espacial de la explotación y la reproducción controlada del sistema. Para los países en vías de desarrollo, la crisis se manifiesta en "los movimientos sociales para la liberación de las estructuras impuestas desde el centro dominante hacia la periferia dependiente, fundamentadas en redes de intercambio desigual y transferencias geográficas del valor" (Soja y Hadjimichalis, 1979, p. 62 [traducción propia]).

Una mirada crítica a la crisis ambiental actual implica reconocer que su solución no proviene únicamente de alternativas tecnológicas, sino de cambios en los estilos de vida de los países industrializados, es decir, cambios hacia una ética para la sustentabilidad, en los modelos productivos y en las técnicas de producción. La crisis debe ser entendida como un problema ético y social, pero, principalmente, político (García Ballesteros, 2000; Edwards, Gil, Vilches y Praia, 2004; Leff, 2006; Casellas, 2008). La causa estructural de la crisis está dada por la racionalidad económica a partir de la cual se administran y gestionan los recursos naturales y por una economía de mercado en la cual están inmersas las formas de administrar y gestionar el territorio que desconocen el funcionamiento y los límites de los sistemas ecológicos. Para el caso de la crisis del agua, la cuestión gira en torno al rol que están teniendo los Estados y los organismos financieros de carácter internacional para impulsar la privatización y los mercados del agua, frente a las demandas de los movimientos sociales que luchan por una gestión democrática y participativa del agua. 
La cuenca del río Sumapaz no ha estado exenta a esta problemática, ya que sus potencialidades ambientales la han convertido en un territorio propicio para el desarrollo de proyectos hidrocarburíferos y minero-energéticos y, a su vez, en un escenario de luchas por la defensa del agua debido a la implantación de dichos proyectos, que favorecen modelos de privatización y ponen en peligro el derecho al agua de las comunidades locales. De esta manera, el propósito principal de este artículo es analizar la resiliencia comunitaria de los actores locales y comunitarios de la cuenca del río Sumapaz a partir de los procesos de defensa del agua y del territorio ocurridos en el periodo comprendido entre los años 2012-2017.

Para mayor claridad en el abordaje y el análisis de la resiliencia comunitaria, el artículo se desarrolla en cinco apartados: el primero se centra en los fundamentos teóricos y conceptuales del tema; el segundo hace referencia a los antecedentes del problema en el contexto colombiano, y el tercero esboza la metodología. Los principales aportes a la discusión se presentan en los apartados cuarto y quinto, en donde se muestran los resultados de la revisión documental y el análisis cualitativo de las entrevistas y las conclusiones del estudio. Cabe anotar que el aporte principal de la investigación a los estudios geográficos se sintetiza en la aproximación que hace al análisis de la resiliencia comunitaria desde una perspectiva geocrítica que involucra el debate actual sobre la geopolítica del agua, en donde se analizan las repercusiones de la implementación de los marcos políticos y económicos internacionales sobre el uso, el acceso y el control del agua; esto ha generado en los actores territoriales ejercicios de democracia deliberativa que reivindican su derecho al agua como un derecho humano fundamental.

\subsection{La crisis del agua, los discursos y los modelos de gestión}

En las últimas cinco décadas, los problemas relacionados con las formas de acceso y distribución del agua en el mundo han llamado la atención de técnicos expertos, líderes comunitarios, políticos y agentes privados, enfocados, sobre todo, en el problema de la escasez que tienen extensas regiones del planeta, como en el caso de África subsahariana, donde más de 300 millones de personas tienen menos de $1 \mathrm{~m}^{3}$ de agua per cápita al año (Organización de las Naciones Unidas para la Educación, la Ciencia y la Cultura [Unesco], 2012).

Otros aspectos como la contaminación de las fuentes superficiales y subterráneas, los estilos de vida de la sociedad moderna y las formas de consumo, el cambio climático y la gestión del agua en el marco de la globalización también configuran la crisis del agua a nivel global. De acuerdo con Vandana Shiva (2003), la escasez del agua en el mundo, de una u otra manera, ha sido provocada por las percepciones y los modos de apropiación y gestión ligados a una determinada cultura del agua, ya sea "una cultura que ve el agua como algo sagrado y considera el suministro como una obligación para preservar la vida y la otra que la considera una mercancía cuya propiedad y comercio son derechos corporativos fundamentales" (p. 10).

En este mismo sentido, cabe mencionar que cada modelo de gestión, sea público, privado o comunitario, está influenciado por una determinada cultura, manifestada a través de discursos que precisan las maneras de pensar y actuar sobre el agua. Esta situación pone en evidencia la complejidad de la gestión en términos de su dependencia respecto de los discursos que manejan los diferentes actores, como expresiones de la cultura del agua que cada 
uno posee. En síntesis, existen dos tipos de discursos: los discursos hegemónicos en la perspectiva de la globalización, provenientes de los gobiernos nacionales, los organismos mundiales de financia-

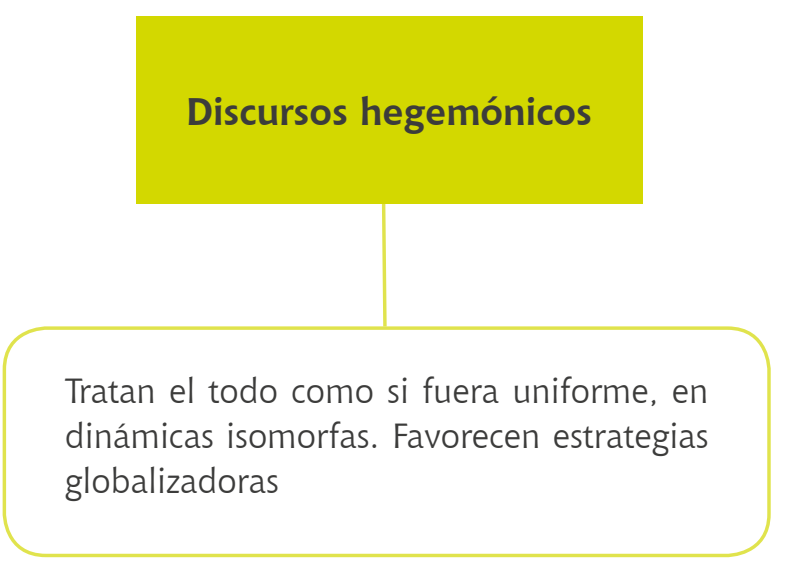

Los discursos de la resistencia abogan por evitar la privatización y el incremento de las tarifas, y son liderados por los gobiernos locales, las comunidades afectadas por la construcción de infraestructura hidráulica y las ONG ambientales; mientras que los discursos de la liberación se centran en la búsqueda de cambios en las políticas y están estructurados en propuestas de cambios sociales, políticos y religiosos resultantes de una nueva sociedad (Vargas, 2006). Valga decir que es precisamente en el marco de los discursos hegemónicos que se han venido favoreciendo los diferentes modelos de privatización del agua.

Otro elemento que se asocia a los discursos del agua es el de las dimensiones o los campos semán- ción, las corporaciones y los académicos y profesionales, y los discursos contrahegemónicos, que se subdividen en discursos de la resistencia y discursos de la liberación (Figura 1).

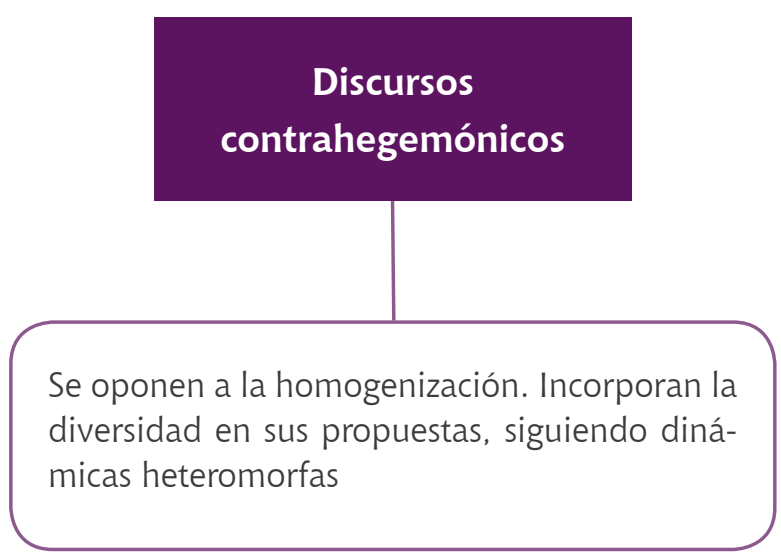

ticos del agua. En este sentido, es posible hablar del agua como elemento vital, como espacio sagrado o como origen de la vida, en la línea del discurso de las sociedades premodernas, o hablar del agua como recurso, como factor de producción o como bien económico, de acuerdo con el discurso de las sociedades modernas.

En consonancia con lo anterior, la Declaración europea por una nueva cultura del agua (Euwater, 2005) propone que es posible identificar por lo menos tres dimensiones del agua que deben tenerse en cuenta en el análisis de los modelos de gestión: el agua-vida, el agua-ciudadanía y el agua-negocio. El agua-vida hace referencia al agua como elemento fundamental para la vida de los seres hu- 
manos y no humanos, la cual debe ser la prioridad y se debe garantizar desde el enfoque de los derechos humanos. Involucra tanto el derecho al acceso al agua salubre como el derecho de las comunidades a la sostenibilidad de los ecosistemas. En el año 2010, la Asamblea de las Naciones Unidas declaró, mediante la Resolución 64/292, el acceso seguro al agua potable y salubre y al saneamiento como un derecho humano fundamental para el completo disfrute de la vida y de todos los demás derechos humanos (Organización de las Naciones Unidas [ONU], 2010).

Una segunda dimensión se refiere al agua-ciudadanía, que está en el segundo nivel de prioridad; comprende los servicios urbanos de agua y las actividades de interés general desde la coherencia de la sostenibilidad. En el tercer nivel de prioridad está el agua como factor de crecimiento económico, el agua-negocio, por el papel que cumple para el desarrollo de las actividades y los negocios privados (Euwater, 2005).

Bajo el enfoque del agua-negocio se sitúan los diferentes modelos de gestión enfocados hacia la privatización, como los principales factores detonantes de la crisis. Estos modelos de privatización se manifiestan por diferentes vías; según Ribeiro (2006), es posible hablar de seis formas de privatización: la privatización de territorios por parte de empresas que utilizan grandes cantidades de agua en sus procesos productivos; la privatización por la contaminación producida por actividades extractivas y de la industria agrícola; los proyectos hidroeléctricos que desvían el curso de los ríos e inundan zonas extensas; la privatización de manantiales por las concesiones que dan los gobiernos a las empresas privadas que venden agua embotellada; la privatización de los servicios de agua potable, saneamiento básico y tratamiento de aguas residuales y, finalmente, la monopolización que hacen las empresas privadas para la extracción de agua subterránea.

La implantación de estos modelos de privatización del agua está generando conflictos sociambientales en diferentes latitudes del planeta. Los conflictos socioambientales son una clase de conflictos ecológicos distributivos que se definen como disputas causadas por el acceso y el control de los recursos (en este caso en particular, del agua). Son procesos sociales suscitados por el desacuerdo que genera la apropiación, la distribución y la utilización de un recurso natural (Sabatini y Sepúlveda, 1997; Martínez-Alier, 2004). En respuesta a estos conflictos socioambientales, las comunidades afectadas se organizan para manifestar su inconformismo y reivindicar su derecho al agua. El ejercicio colectivo para la defensa del agua es un ejemplo claro de las luchas que se viven hoy en los territorios, en el marco de los discursos contrahegemónicos de la resistencia que le hacen frente a las diferentes formas de producción territorial impuestas por las políticas de la globalización.

\subsection{La resiliencia comunitaria y la defensa del agua y el territorio}

La resiliencia comunitaria es concebida como una práctica social que ha sido estudiada como un proceso dinámico de adaptación exitosa a la adversidad (Reich, Zautra y Hall, 2010), producto de la crisis ambiental global. Se caracteriza por incorporar el análisis de las condiciones colectivas que tienen los grupos humanos para hacerle frente a los desastres (Suárez, 2001; Folke, 2006; Maguire y Cartwright, 2008). Los recursos con los que cuenta 
una comunidad, su capacidad de adaptación y su capacidad de asimilar los disturbios, son elementos fundamentales de la resiliencia comunitaria; es "la capacidad de una comunidad para utilizar sus recursos con el fin de adaptarse a una adversidad, y eventualmente ser capaz de absorber la perturbación, volver a la rutina e incluso desempeñarse mejor en comparación con la situación previa a la perturbación” (Rapaport et al., 2018, p. 471 [traducción propia]) lo que identifica a una comunidad como resiliente. Para Uriarte (2013), estas mismas características definen la resiliencia en una perspectiva de transformación, en la cual es la capacidad de las personas para responder y adaptarse a los cambios a través de su reorganización la que les permite generar oportunidades nuevas a partir de la crisis, fortaleciéndose y transformándose positivamente desde la experiencia vivida.

Para los autores de este artículo, la resiliencia comunitaria es una característica de las poblaciones que han resistido condiciones sociales, ambientales, económicas y políticas adversas que los han marginado de un desarrollo equitativo, pero que, al mismo tiempo, les ha dado la fuerza para sobreponerse a situaciones desfavorables, fortaleciendo su identidad cultural y su capacidad de organización solidaria alrededor de sus necesidades más sentidas. Ahora bien, desde la teoría social y en el contexto latinoamericano, los principales pilares que identifican a las comunidades resilientes son la identidad cultural, la autoestima colectiva y la cohesión social (Suazo, 2016). La identidad cultural puede ser comprendida a partir de los comportamientos, los valores, las creencias y las costumbres compartidas por la comunidad, que generan un sentido de pertenencia y refuerzan las actitudes de solidaridad (Suárez, 2001; Uriarte, 2013); im- plica que tanto los individuos como las comunidades se reconocen históricamente, en su entorno físico y social (Molano, 2007).

La autoestima colectiva, por su parte, reúne elementos relacionados con la actitud y el sentimiento de orgullo por el lugar donde se vive, el amor por su tierra, la conciencia de las bellezas naturales del lugar (Uriarte, 2013), así como el orgullo por la comunidad misma (Suárez, 2001); es una característica diferencial de los individuos que puede ayudar a mantener una identidad social positiva (Crocker y Luhtanen, 1990). La cohesión social hace referencia al poder de la acción conjunta, la eficacia colectiva, las actividades solidarias al interior de una comunidad y sus experiencias de autoorganización (Uriarte, 2013). Otra característica importante de la resiliencia comunitaria son los ejercicios de democracia participativa, en los que los ciudadanos participan directamente en la toma de decisiones estatales y tienen injerencia directa en la planificación, la ejecución y el control de la gestión pública, lo cual alude a una democracia más participativa que representativa. Tal como afirma Uriarte (2013), "una sociedad es resiliente cuando ejerce la democracia participativa y exige la transparencia en la gestión pública” (p. 16).

Los procesos de defensa del agua y del territorio que se generan a partir de la implantación de proyectos minero-energéticos en América Latina, en el marco del discurso del crecimiento verde, son una manifestación de la resiliencia comunitaria, en la medida en que reúnen un conjunto de sentidos, contenidos, prácticas y tradiciones de las comunidades (Úcar, 2009) que les permite fortalecer su apropiación del espacio y la construcción del territorio, así como el entendimiento crítico de las problemáticas y los conflictos para fortalecer su 
rol como agentes de cambio a través de una toma de conciencia sobre el ser, el estar y el actuar en el territorio.

\subsection{La defensa del agua y del territorio en Colombia}

Colombia, ubicado en la parte noroeste de Suramérica, es un país con una tradición importante en la defensa del agua y del territorio: desde comienzos del siglo pasado se han llevado a cabo movilizaciones sociales y protestas a nivel nacional por la falta de acceso al agua y de sistemas de acueducto apropiados para abastecer a la población, sobre todo de las áreas rurales. La ampliación de la cobertura, el mejoramiento de la calidad y el sistema tarifario también han estado en la lista de las demandas de la población; de allí que la gestión comunitaria para el abastecimiento de agua sea vista como una respuesta a la ausencia del Estado, y estas demandas se evidencian en las protestas sociales durante el último siglo (Gómez, 2014).

Un proceso significativo de defensa del agua en Colombia es la propuesta del referendo por el agua que adelantó el Comité Nacional de Defensa del agua y de la Vida en el año 2007, a partir de la iniciativa de la Defensoría del Pueblo y de Ecofondo. El referendo proponía una reforma a la Constitución de 1991 para declarar el agua como derecho humano fundamental, lo cual implicaría que el Estado estaría en la obligación de suministrar agua potable a todas las personas y de garantizar un mínimo vital gratuito; consagrar el agua, en todas sus formas y estados, como parte constitutiva de los territorios colectivos de indígenas y comunidades negras, y como elemento sagrado en la cosmovisión de los grupos étnicos; el compromiso del Estado de proteger los ecosistemas esenciales para el ciclo del agua, y de procurar la implantación de modelos de uso sustentable para garantizar la disponibilidad del líquido en el futuro. Desafortunadamente, en el mes de diciembre de 2009 la iniciativa inicial se hundió en segundo debate de la Comisión Primera de la Cámara de Representantes; en el último cuatrienio (2014-2018), la iniciativa se retomó con el apoyo del Partido Verde, sin embargo, al finalizar el primer periodo de la tercera legislatura (diciembre de 2016) se hundió nuevamente por no alcanzar a tener siete de los ocho debates necesarios para su aprobación. Esta situación muestra cómo este no es un tema prioritario para los actores políticos que nos representan en el Congreso de la República.

En los últimos diez años también se han presentado diferentes movilizaciones en el país en defensa y protección del agua, especialmente en contra de la actividad minera y la exploración y explotación de hidrocarburos que se desarrollan en zonas de páramo, ecosistemas estratégicos que brindan el agua a grandes sectores de la población, tanto urbana como rural. Tal es el caso del municipio de Bucaramanga con el páramo de Santurbán, en el departamento de Santander, y los municipios de Cajamarca, Piedras e Ibagué en el departamento del Tolima. Se trata de procesos participativos enmarcados en lo que Roa García, Roa Avendaño y Acosta (2017) han denominado "La ola de democratización ambiental en Colombia” (p. 331).

A partir de este contexto, se escogió como área de estudio de esta investigación la cuenca del río $\mathrm{Su}$ mapaz, ya que en su parte alta se encuentra el páramo de Sumapaz, una de las reservas hídricas más grandes del país. Los ríos y quebradas que nacen en el páramo abastecen los acueductos de los municipios de la provincia de Sumapaz, el municipio 
de Icononzo en el departamento del Tolima y el corregimiento de San Juan de Sumapaz en la localidad 20 de Bogotá. En la cuenca hay presencia de corredores biogeográficos y áreas protegidas que la definen como ruta óptima para establecer la red de conectividad ecológica regional (Corporación Autónoma Regional de Cundinamarca, 2012). La presencia de proyectos de generación de energía (proyecto hidroeléctrico El Paso, de la empresa Emgesa S.A. E.S.P) y de exploración petrolera (área contratada para la exploración de hidrocarburos Bloque COR 4 adjudicado en el año 2010 a la empresa Australian Drilling Associates Pty Ltd. Sucursal Colombia) han suscitado la emergencia de conflictos socioambientales por el agua entre los actores locales (principalmente las asociaciones de acueductos comunitarios) y el gobierno nacional, quien ha entregado el aval para dichos proyectos.

\section{Metodología}

Para el reconocimiento de los procesos de participación comunitaria en defensa del agua y del territorio en la cuenca del río Sumapaz se realizó una revisión documental y trabajo de verificación en campo para indagar con los diferentes actores claves del territorio. Para la revisión documental se escogieron informes y documentos de institu- ciones como autoridades ambientales, autoridades de vigilancia y control e instituciones que manejan la información ambiental, social y estadística del país. Se revisaron 20 documentos, entre libros y artículos científicos de bases de datos como SciELO, RedALyC, Scopus, la Red de Bibliotecas Virtuales de Consejo Latinoamericano de Ciencias Sociales (Clacso) y del Instituto Latinoamericano para una Sociedad y un Derecho Alternativos (ILSA), así como la biblioteca del Instituto Colombiano de Antropología e Historia (ICANH) y la Biblioteca Luis Ángel Arango de Bogotá. Los criterios de búsqueda se centraron en las categorías Sumapaz, conflictos y defensa del agua en los últimos cinco años, aunque para comprender el contexto histórico se revisaron también documentos publicados entre 1990 y 2017. Para identificar las actuales manifestaciones de resiliencia comunitaria en la cuenca del río Sumapaz se aplicó una entrevista semiestructurada en el mes de julio de 2017 a 20 actores territoriales clave. En la Tabla 1 se relaciona el número de actores entrevistados y la organización a la que pertenecen. Las cuatro preguntas analizadas de la entrevista fueron: ¿qué aspectos conoce acerca de la gestión del agua en el municipio? ¿Cuáles son los principales problemas de la gestión del agua? ¿Qué opina sobre los conflictos relacionados con el agua que existen hoy en el Sumapaz? ¿Ha participado en la búsqueda de alternativas de solución de estos conflictos? 


\begin{tabular}{l} 
TABLA 1. NÚMERO DE ACTORES ENTREVISTADOS \\
\hline \multicolumn{1}{c}{ Organización } \\
\begin{tabular}{lc}
\hline Alcaldía Municipal & Número de actores \\
\hline Empresa de servicios públicos & 2 \\
\hline Asociación de acueductos & 5 \\
\hline Representantes del Concejo Municipal, & Subcomarca \\
\hline juntas de acción local o juntas de acción comunal & (Paraje simple) \\
\hline Academia & 4 \\
\hline Fuente: Elaboración propia & 2 \\
\hline
\end{tabular}
\end{tabular}

Para el análisis de las entrevistas se utilizó el software MAXQDA 12, especializado en análisis cualitativo, que permite sintetizar las percepciones de los diferentes actores territoriales y realizar el análisis categorial de las mismas. Las categorías escogidas para el análisis de la resiliencia comunitaria en la cuenca fueron la identidad cultural, la autoestima colectiva, la cohesión social y las prácticas de democracia participativa, que se definen como pilares fundamentales en las comunidades resilientes.

\section{Resultados y discusión}

Los resultados se presentan en dos partes: la primera corresponde a la información general del territorio donde se desarrolló la investigación e incluye una síntesis del contexto histórico y los conflictos socioambientales actuales, así como de los procesos de participación en torno a la defensa del agua; esto a partir de la revisión documental y su correspondiente análisis, realizado de acuerdo con los parámetros explicados en la metodología. La segunda parte presenta el análisis de las entrevistas, para comprender las percepciones de los actores clave según las categorías establecidas en la metodología.

\subsection{La participación comunitaria en defensa del agua y del territorio en la cuenca del río Sumapaz}

La cuenca del río Sumapaz está localizada en la Región Andina colombiana, al suroccidente de la ciudad de Bogotá. Tiene una extensión de $3.048,32 \mathrm{~km}^{2}$ y está integrada por los municipios de Granada, Silvania, Fusagasugá, Pasca, Tibacuy, Arbeláez, San Bernardo, Cabrera, Venecia, Pandi y Nilo, en el departamento de Cundinamarca, los municipios de Icononzo, Melgar y Cármen de Apicalá en el departamento del Tolima, y el corregimiento de San Juan de Sumapaz, en zona rural del Distrito Capital. La cuenca está 
dividida en 12 microcuencas que corresponden a los ríos Analí, Juan López, Quebrada Negra, Alto, Medio y Bajo Sumapaz, Negro, Pagüey, Panches,
Pilar y San Juan (Corporación Autónoma Regional de Cundinamarca, 2007), como se muestra en la Figura 2.

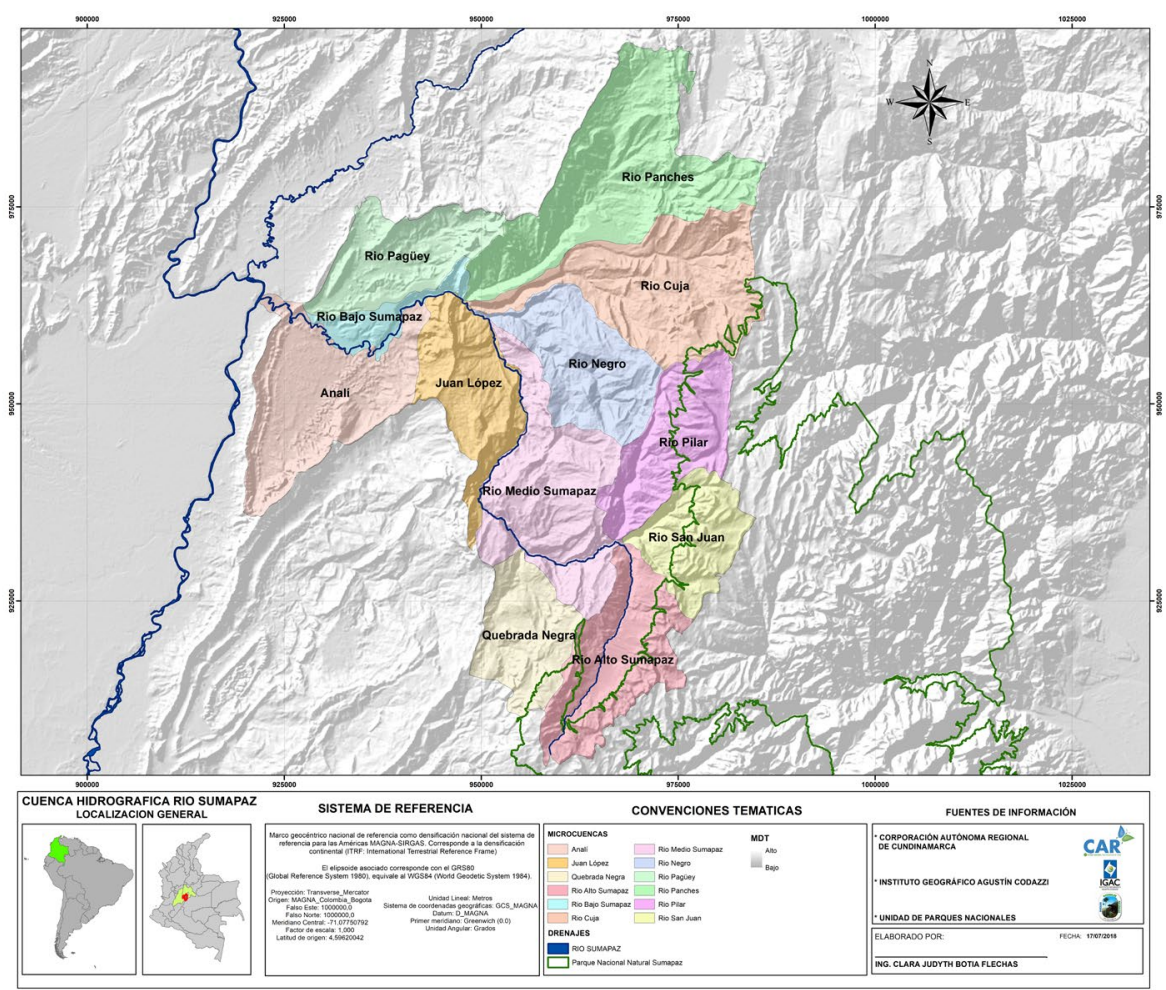

FIGURA 2.
Modelo digital de la cuenca
del río Sumapaz.
Fuente: Elaboración propia con base en la información oficial del Ministerio de Ambiente y Desarrollo Sostenible, el Sistema de Parques Nacionales Naturales y la CAR. Base cartográfica del Instituto Geográfico Agustín Codazzi.
Según las proyecciones del Departamento Administrativo Nacional de Estadística (DANE), con año de referencia del Censo 2005, para el año 2015 la población de la cuenca era de 295.108 habitantes. En la parte suroriental de la cuenca se encuentra un sector del páramo de Sumapaz que pertenece al Parque Nacional Natural de Sumapaz, una de las reservas de agua más importantes del país. La cuenca hace parte de la jurisdicción de la Corporación Autónoma Regional de Cundinamarca (CAR), la Corporación Autónoma Regional del Tolima (Cortolima) y la Unidad Administrativa de Parques Nacionales Naturales.
Desde la década de 1930 el territorio de la cuenca ha presentado cambios en la organización espacial a causa de procesos de colonización, formas en la tenencia de la tierra (minifundio) y actividades agrícolas y ganaderas, trasformaciones que estuvieron enmarcadas en un contexto de conflicto armado debido al accionar de frentes de la guerrilla de las FARC, grupos de Autodefensas y operaciones militares de control, especialmente en las décadas de 1980, 1990, 2000 y 2010. De otro lado, las potencialidades ambientales han convertido este territorio en un lugar propicio para el desarrollo de proyectos hidroeléctricos y mineros. 
Tradicionalmente esta región ha estado sometida a procesos de lucha por la tierra y allí se han gestado movimientos sociales importantes (Marulanda, 1991), sin embargo, en la actualidad las luchas en la cuenca del río Sumapaz son por la defensa del agua y la defensa del territorio.

Como se mencionó, los conflictos socioambientales por el agua de la cuenca han emergido en los últimos diez años y están relacionados con la ejecución de dos proyectos, uno de generación de energía y el otro de exploración y producción de hidrocarburos; ambos proyectos están avalados por el Gobierno nacional. El proyecto hidroeléctrico se denomina El Paso y está liderado por la multinacional Emgesa. Los principales efectos que se generarán con su construcción en la cuenca del río Sumapaz se relacionan con la contaminación del agua y cambios en la potabilidad, la variación en los ciclos naturales del caudal (por el desvío del río) y la disminución en la fertilidad del suelo y la producción agrícola. Hoy en día las comunidades que habitan las zonas afectadas no tienen claridad sobre todas las obras que se van a ejecutar ni sobre los impactos que se pueden producir con su construcción, por este motivo están adelantando estrategias de reapropiación del territorio para aumentar su conocimiento sobre la problemática socioambiental del proyecto y generar procesos de movilización social en defensa del páramo y el recurso hídrico como pilares de la economía campesina (León y Villarraga, 2014).

En relación con el proyecto de exploración y producción de hidrocarburos, actualmente se está desarrollando la fase exploratoria del contrato Bloque COR 4 en los municipios de Fusagasugá, Pandi y Pasca a cargo de la empresa Australian Drilling Associates, así como las exploraciones en los mu- nicipios de Arbeláez, San Bernardo e Icononzo por la compañía Alange Energy y la empresa nacional Vector (Roa, 2014). Frente a esta situación, las comunidades organizadas han desarrollado una serie de acciones para manifestar a los entes gubernamentales y a la opinión pública en general su inconformismo con dichos proyectos, cuestionando las decisiones del Gobierno de otorgar títulos mineros y licencias de construcción de los proyectos en áreas estratégicas de reserva hídrica.

Las organizaciones de usuarios de los acueductos comunitarios, tanto para consumo humano como para riego, son quienes vienen liderando estos procesos de protesta y movilización para la reivindicación de su derecho al territorio al que han pertenecido ancestralmente y con ello el derecho al agua, en un ejercicio de democracia participativa que es ejemplo para el país. De acuerdo con la Contraloría de Cundinamarca (2016), actualmente existen en la cuenca del río Sumapaz 143 acueductos rurales que brindan el agua a 37.757 usuarios y benefician una población aproximada de 151.028 personas.

Las principales acciones que han desarrollado estas organizaciones son las movilizaciones, como la Caravana en Defensa del Agua y el Territorio, desde el municipio de Fusagasugá hasta la plaza de Bolívar en Bogotá, el 16 de septiembre de 2014, donde los delegados de la comunidad radicaron un derecho de petición respaldado por 25.000 firmas de los habitantes de la región para que el presidente de la república, Juan Manuel Santos, frenara los proyectos de explotación de hidrocarburos y proyectos mineros en el páramo (Romero, 2014).

También se ha hecho uso de mecanismos de participación sistémica, como las consultas populares, para que las personas voten si desean o no 
la explotación de petróleo y el desarrollo de proyectos hidroeléctricos en su municipio. El tema de las consultas populares inició en el mes de febrero de 2017, cuando el municipio de Cabrera, con el $97 \%$ de votos, le dijo no a la pregunta: ¿Está usted de acuerdo, sí o no, con que en el municipio de Cabrera, Cundinamarca, como Zona de Reserva Campesina, se ejecuten proyectos mineros y/o hidroeléctricos que transformen o afecten el uso del suelo, el agua o la vocación agropecuaria del municipio? Lo propio sucedió el
9 de julio en el municipio de Arbeláez, donde 4.312 de los 4.376 votos registrados fueron por el no a la pregunta: ¿Está usted de acuerdo, sí o no, con que en la jurisdicción del municipio de Arbeláez, Cundinamarca, se lleven a cabo procesos de sísmica, exploración, explotación de hidrocarburos, lavado de hidrocarburos y minería de gran escala? (Roa García et al., 2017). Otros municipios, como San Bernardo y Pasca, están en el proceso para la realización de la consulta (Figura 3).

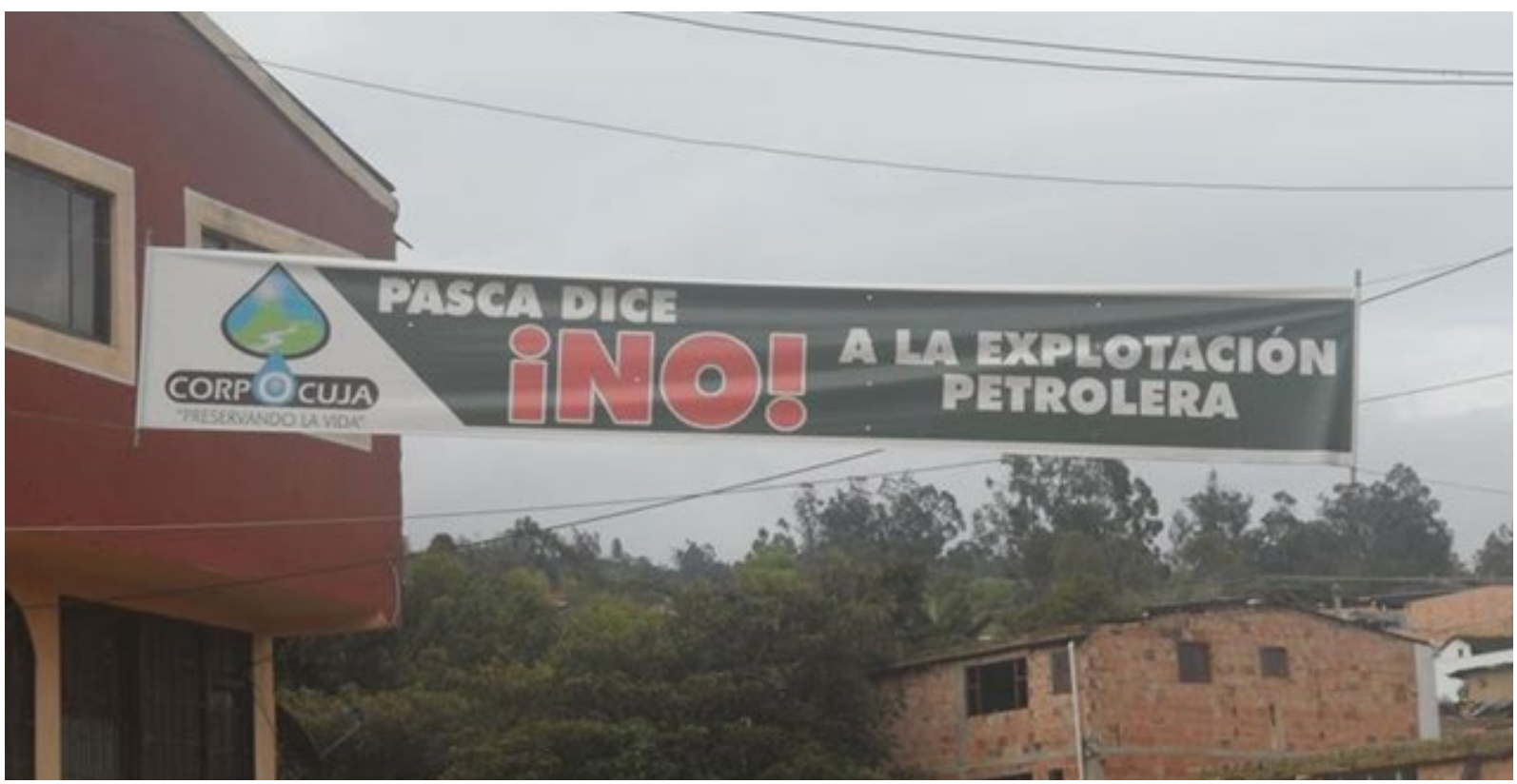

\section{FIGURA 3.}

Difusión de la consulta popular en el municipio de Pasca. Fuente: Los autores (2017).

El otro instrumento ha sido el de las tutelas que se han instaurado por el derecho a gozar de un ambiente sano y el derecho a la vida, en relación con el derecho humano al agua. En marzo de 2017 se radicaron 2.376 tutelas: 190 en Icononzo (Tolima), 42 en San Bernardo, 621 en Pandi, 1.200 en Arbeláez y 323 en Fusagasugá.
En los meses de junio y julio del año 2017 también se realizaron movilizaciones y bloqueos en la parte alta de la cuenca, en inmediaciones de la laguna de Chisacá, pero esta vez con el propósito de frenar el acceso indiscriminado de turistas al área del páramo de Sumapaz, que hace parte del Parque Nacional Natural del Sumapaz. Esto con el fin de evitar 
el deterioro del lugar por el uso inadecuado que están haciendo los turistas de esta área protegida.

\subsection{La resiliencia comunitaria en la cuenca del río Sumapaz}

En el territorio de la cuenca del río Sumapaz, la resiliencia comunitaria tiene una tradición que es importante mencionar aquí: las comunidades que habitan la cuenca han estado históricamente envueltas en escenarios de luchas sociales para reivindicar su derecho al territorio. A comienzos del siglo pasado las luchas eran por la tierra, ya que la estructura espacial de grandes haciendas cafeteras generó condiciones de injusticia e inequidad social entre hacendados, colonos y arrendatarios. Frente a esta situación, las comunidades resistieron, se organizaron y conformaron el primer sindicato agrario del país (Marulanda, 1991; Varela y Duque, 2011).

Estas mismas comunidades resistieron el embate del conflicto armado que durante cuatro décadas

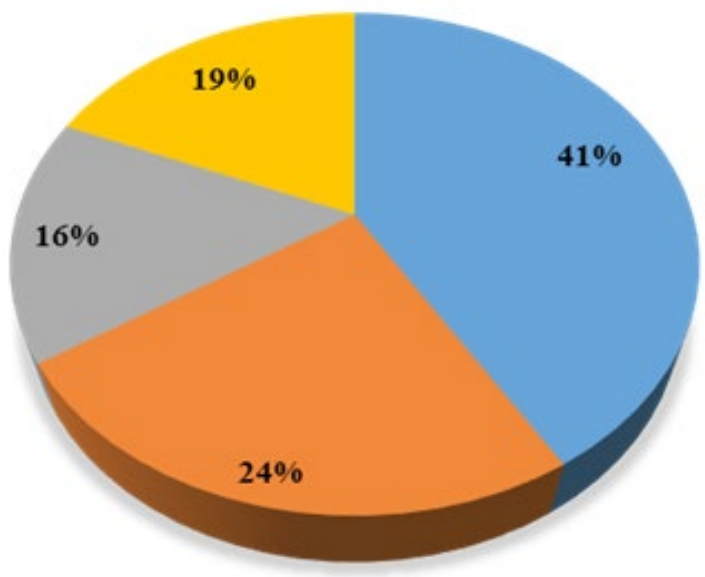

los afectó, debido los enfrentamientos entre la fuerza pública, la guerrilla y los grupos de autodefensas, así como los secuestros y las extorsiones a agricultores, empresarios y comerciantes de la región (Vicepresidencia de la República de Colombia, Observatorio del Programa Presidencial de Derechos Humanos y Derecho Internacional Humanitario, 2002). En la actualidad los habitantes de la cuenca están organizados alrededor de la defensa del agua y del territorio para hacerle frente a las políticas económicas y los modelos de privatización del agua, en un ejercicio de democracia deliberativa del cual la academia tiene mucho que aprender.

El análisis de las entrevistas realizadas a los actores clave evidencia diferentes manifestaciones en relación con la resiliencia comunitaria. A partir de las respuestas obtenidas se codificaron 70 segmentos $^{3}$, de los cuales el $41 \%$ está asociado a la categoría autoestima colectiva y el $24 \%$ a la categoría cohesión social. En la Figura 4 se muestra la distribución de los segmentos por cada categoría.
FIGURA 4.

Distribución de las categorías de análisis. Fuente: Elaboración propia.

3 Los segmentos son frases o párrafos seleccionados de las respuestas textuales de los actores entrevistados que dan cuenta de las categorías de análisis. 
La autoestima colectiva se ve reflejada en diferentes expresiones de los actores territoriales donde se manifiesta su interés por la defensa del territorio frente a las actividades de exploración y explotación de hidrocarburos, así como en una conciencia colectiva sobre la importancia del agua como patrimonio natural de la región, ligada a una identidad cultural en torno al páramo de Sumapaz y al agua.

Estamos amenazados por un proyecto de hidrocarburos que se llama Cordillera Cuatro, donde está Fusagasugá, Sibaté, Arbeláez, Pasca, San Bernardo, y el sector donde tenemos la captación está incluido en los polígonos de este proyecto. Si se llegase a dar afectaría mucho todo el ecosistema. Con la riqueza del agua, acá sabemos cuál es el impacto, sería algo ilógico que no defendiéramos nuestro páramo, para nosotros el agua es la vida y si no la defendemos estamos llevados (Entrevista a un representante de una asociación de acueductos, municipio de Fusagasugá, 2017).

Asimismo, los actores reconocen su preocupación por la explotación de petróleo en el territorio y los impactos sociales y ambientales que esto trae consigo.

Eso es grave, y fuera del daño ecológico que hacen, porque ellos acaban, absorben el suelo y acaba con el suelo, entonces dejan el suelo estéril, eso ya no sirve para nada, desvalorizar las tierras, todo eso es malo y donde va uno a mirar, vaya, mire, hago un recuento de todas las zonas donde han explotado petróleo y verán que eso está acabado, ya nadie quiere comprar nada (Entrevista a un funcionario de la Alcaldía, municipio de Fusagasugá, 2017).

También en las palabras que revelan un sentimiento de orgullo por el territorio donde se vive y en la importancia de defender ese territorio:

Pues está la minería, pues que dice el Gobierno que ellos son dueños del subsuelo, pero entonces, pues nosotros somos los que estamos viviendo en el suelo, entonces si acaban el subsuelo se va a acabar el suelo, entonces, pues yo no estoy de acuerdo con eso porque nosotros sí hemos vivido los cambios y los efectos de la explotación, pues cuando ellos venían a hacer una reunión es como concientizando la población de que eso no pasaba nada, y entonces yo siempre les alegaba, yo siempre decía que no podemos permitirlo porque nos van a acabar con el agua (Entrevista a un funcionaria de la Alcaldía, municipio de Pandi, 2017).

Se manifiesta, del mismo modo, en la satisfacción por la respuesta de la comunidad a las acciones colectivas emprendidas para la defensa del agua y del territorio.

Hicimos una tutelatón, un domingo de tutelas, y teníamos programado sacar 200 tutelas, pero la gente respondió mucho y conseguimos 700 tutelas en todo el municipio, y la gente se moviliza mucho frente al tema porque el tema es delicado para nosotros (Entrevista a un integrante de una Junta de Acción Comunal [JAC], municipio de Pandi, 2017).

En síntesis, se manifiesta una conciencia sobre habitar (una conciencia del estar) en inmediaciones 
del páramo de Sumapaz, considerado como una reserva hídrica muy importante; una conciencia colectiva crítica de la problemática de los conflictos socioambientales en el territorio; también se evidencia el compromiso que tienen con su territorio y la apropiación de sus problemáticas y conflictos, lo que otorga una significativa relevancia a las acciones colectivas para la conservación, que fortalecen el orgullo por su territorio y su comunidad.

Con respecto a la cohesión social, los actores territoriales expresaron algunos aspectos como la relevancia de la organización comunitaria (procesos de autoorganización) en la defensa del agua y del territorio.

Acá se creó una corporación que la componen las asociaciones de acueductos y de distritos de riego que toman agua del río Cuja, Corpocuja, que está liderando una consulta popular para "no a la explotación petrolera" (Entrevista a un representante de una asociación de acueductos, municipio de Pasca, 2017).

Hay un interés por un grupo grande que se abrió un espectro donde los sectores se han unido, manejando un colectivo por el agua que se llama Agua Sí, Petróleo No, entonces, yo digo, excelente, y ya no es solo una voz, sino hay muchas voces por ahí moviéndose, y están haciendo, y estamos mirando la posibilidad para que en Fusagasugá logremos ir a las urnas y decir no a la explotación (Entrevista a un concejal del municipio de Fusagasugá, 2017).

También se evidencia que la comunidad está de acuerdo con proteger el territorio de la explotación petrolera y las hidroeléctricas. Hay una conciencia crítica de la problemática actual del territorio, que, asimismo, se relaciona con la autoestima colectiva.

Supuestamente ellos dicen [las petroleras] que el impacto ambiental, que no va a haber, pero siempre hay impacto ambiental, sea el más mínimo que sea, lo va a haber. Entonces, nosotros siempre hemos tenido desconfianza sobre eso, y aquí la mayoría de las personas tienen desconfianza sobre eso. Sea lo del petróleo, lo que dicen los muchachos, vienen esas compañías, sacan todas nuestras riquezas y después de quince, veinte años nos dejan en la nada. Entonces, aquí hay mucha oposición, también en el pueblo, sobre eso (Entrevista a un representante de una JAC, municipio de Pandi, 2017).

De igual modo, la cohesión social se expresa en la participación de las asociaciones de acueductos y de la población en general en la defensa del agua y del territorio.

Se vinculó informándole a la comunidad, tuvimos unas asambleas justamente en la época del permiso para realizar la consulta aquí, aquí no queremos la explotación minera, no la queremos, precisamente por eso solicitamos la consulta (Entrevista a un representante de una asociación de acueductos, municipio de Pasca, 2017).

Nosotros siempre hemos tenido una política aquí, de hecho, yo he participado marchando, porque nosotros estamos es a favor del agua y en contra de las petroleras (Entrevista a un edil del municipio de Fusagasugá). 
Asimismo, se manifiesta a través de acciones conjuntas para la protección del territorio:

Precisamente el domingo pasado estuvimos en un plantón en la frontera entre la localidad de Usme y la localidad de Sumapaz. ¿Qué objetivo tenía este plantón? De la mejor manera, hacerle entender al turista que no se le permite el ingreso (Entrevista a un docente de la localidad de $\mathrm{Su}$ mapaz, 2017).

Se evidencia una gestión participativa en torno al cuidado del agua, la protección de las fuentes, la conservación de los bosques y las experiencias de autoorganización que emergen alrededor de un tema fundamental para la comunidad. De esta manera, es en la organización de las comunidades y su participación en las acciones colectivas para la defensa y la protección de la cuenca en donde mejor se expresa un sentido de cohesión social en el territorio.

Sobre la identidad cultural, las entrevistas revelan que está presente en relación con el páramo y la región del Sumapaz:

Somos la mata del agua del mundo, somos el pulmón del mundo, el más extenso del mundo, las tierras de estas zonas son unas de las más fértiles, tenemos una producción agropecuaria de toda la zona Arbeláez, San Bernardo, Cabrera, Silvania, Sibaté, toda esta zona del Sumapaz. Es una extensa zona que abastece gran parte de Corabastos en Bogotá. Entonces, si se explota, eso los perjuicios van a ser más grandes que los beneficios (Entrevista a un representante de una asociación de acueductos, municipio de Pasca, 2017).
La región del Sumapaz se ha destacado por ser una reserva campesina muy importante porque [de] aquí salen muchos productos, tanto para Bogotá como para esta región, entonces me parece importante que la gente piense en el campo porque es a donde tenemos que volver (Entrevista a un estudiante universitario del municipio de Fusagasugá, 2017).

La identidad cultural también se ve reflejada en los esfuerzos para activar el sentido de pertenencia y los lazos de solidaridad:

La consulta popular en Fusagasugá, aunque es necesaria, sabemos que es muy difícil de manejar, estamos tratando de concientizar a los fusagasugueños de que lo que pasa en Pasca nos compete a todos, es que desconocemos de dónde viene el agua (Entrevista a un representante de una asociación de acueductos, municipio de Fusagasugá, 2017).

Por eso que tenemos ese proyecto de la cultura ambiental rural dirigida a todos, pero especialmente a los niños, porque la primera forma de ataque ante todas esas cosas es que todos los ciudadanos, cuando estén más grandes, sean conscientes de que cada cosa mal que hacen contra el medio ambiente es un pecado contra la humanidad (Entrevista a un integrante de una JAC, municipio de Fusagasugá, 2017).

En resumen, un elemento clave de la identidad cultural en los actores entrevistados se relaciona con el significado que tiene para ellos habitar un ecosistema como el páramo de Sumapaz, al rededor del cual se comparten valores, creencias y costum- 
bres sobre el agua que fortalecen su conciencia del ser y del estar.

En relación con las prácticas de democracia participativa, los actores territoriales manifiestan la importancia del mecanismo de consulta popular para la defensa del agua y del territorio:

Aquí ya está concesionado por Canacol Energy una zona, porque ya hicieron la sísmica y efectivamente hay petróleo $\mathrm{y}$ están tramitando la licencia ambiental con la ANLA, ahí es donde nosotros, con la consulta popular, los frenamos (Entrevista a un representante de una asociación de acueductos, municipio de Pasca, 2017).

Yo he visto con mucha alegría y me ha causado mucha emoción ver que la gente se ha organizado para a través de acciones electorales decir no queremos, acá, por ejemplo, la explotación minero energética, y he visto en municipios donde se ha hecho esa consulta y ha ganado el no, no queremos; afortunadamente tenemos esa herramienta (Entrevista a un concejal del municipio de Fusagasugá, 2017).

Pues nosotros estamos de acuerdo con la posición del municipio, la realización de la consulta popular sobre la minería en el municipio y demás, y obviamente nosotros tenemos que avalar el proceso porque es el mejor mecanismo para la protección del recurso hídrico (Entrevista a un representante de una empresa de servicios públicos, municipio de Fusagasugá, 2017).

El objetivo es darnos la pela [con el agua] hasta lo máximo... Cabrera, por ejemplo, ya hizo la consulta popular, entró en pro- ceso Arbeláez, que la hace en el mes de julio, en agosto la va a hacer el municipio de Pasca. Posiblemente nosotros la estamos realizando entre octubre y noviembre, es prácticamente la prioridad y es el debate en el que estábamos hoy con el comité de veeduría y el concejo municipal, para que nosotros blindemos prácticamente la riqueza hídrica de nuestro país y de nuestro municipio (Entrevista a un funcionario de la Alcaldía del municipio de San Bernardo, 2017).

También se evidenció el trabajo de la academia que apoya los procesos de democracia participativa:

Pues nosotros, desde el semillero, hemos estado en las consultas, entonces hemos apoyado, estuvimos en lo último de $\mathrm{Ca}$ brera, estuvimos en un foro de Pandi que fue superimportante porque se reunieron varios grupos de campesinos en ese lugar y nos contaron, digamos, las vivencias que tenían ellos y todo lo que estaba pasando, y estuvimos en Arbeláez apoyando toda la consulta y apoyando sobre todo a Huacana, que era uno de los primordiales que estaba ahí, es un colectivo que se llama Huacana y ahorita estamos apoyando el de Pasca (Entrevista a un estudiante universitario, municipio de Fusagasugá, 2017).

Los procesos de democracia participativa que se vienen desarrollando en el territorio de la cuenca, como movilizaciones, tutelas y consultas populares (mencionadas anteriormente), son, sin lugar a duda, uno de los pilares fundamentales de la resiliencia comunitaria en el Sumapaz. Esta región viene dando pasos importantes en la construcción 
de procesos comunitarios a partir de ejercicios de democracia participativa que han determinado la toma de decisiones y posibilitarán, en un futuro inmediato, una organización comunitaria madura y coherente que permitan la defensa de los intereses colectivos, especialmente aquellos ligados al uso y la conservación de los recursos naturales.

\section{Conclusiones}

Las preocupaciones de las comunidades por lo que pueda pasar con el agua en su territorio debido a los proyectos de explotación minera y de generación de energía hidroeléctrica se materializan en acciones concretas de democracia participativa y dan cuenta del sentido de apropiación de estos grupos humanos por su territorio. La resiliencia comunitaria de los habitantes de la cuenca le otorga una identidad y un sello particular a la territorialidad que allí expresan.

Existe una conciencia colectiva crítica de la problemática de los conflictos socioambientales en el territorio, que evidencia el compromiso que tienen con su territorio y la apropiación de sus problemáticas y conflictos. Sin duda, son comunidades que mantienen una identidad cultural, una autoestima colectiva y una cohesión social alrededor de sus realidades sociales, económicas y ambientales.

Los procesos de defensa del agua y del territorio en la cuenca del Sumapaz han tenido una tradición histórica y se han constituido en una parte de la identidad de sus pobladores. Las diferentes adversidades por las que han tenido que pasar estas comunidades les han permitido reorganizarse para convertirse en agentes de cambio que se apropian cada vez mejor de sus realidades territoriales. De esta manera, la resiliencia comunitaria ha estado y está presente en la defensa del agua y del territorio, en la medida en que estos procesos revelan una cohesión social que permite la participación efectiva de los actores locales y la ejecución de acciones conjuntas y solidarias que reivindican la identidad cultural de las comunidades de la cuenca.

Los actores locales y comunitarios trabajan en la lógica de la justicia ambiental y la justicia hídrica, en un discurso contrahegemónico que se enfoca en la dimensión del agua-vida como la prioridad, que busca el reconocimiento del papel legítimo que tienen para reivindicar su derecho humano al agua, proteger las fuentes locales de agua y garantizar el abastecimiento, bajo un modelo de gestión solidaria y cooperativa del agua.

Existe una relación intrínseca entre las categorías de análisis de la resiliencia comunitaria. Así, por ejemplo, cuando las comunidades tienen un arraigo por su identidad cultural, esto fortalece la autoestima colectiva y la cohesión social, y esta última es la que, a su vez, facilita los ejercicios de democracia participativa en el territorio.

Esta capacidad de estas comunidades para organizarse y enfrentar las adversidades en una apropiación social y cultural consciente del espacio, de sus sistemas naturales y de sus problemáticas, es una cualidad que debe ser reconocida por parte de los actores institucionales del nivel local y nacional para la ejecución de una planificación participativa orientada a la sostenibilidad territorial de la cuenca. 


\section{Referencias}

Casellas, A., (2008, mayo). Geografía eco-crítica: el giro medioambientalista como eje vertebrador de una nueva territorialidad. Ponencia presentada en el X Coloquio Internacional de Geocrítica, Diez años de cambios en el mundo, en la geografía y en las ciencias sociales, 1999-2008, Barcelona, España.

Contraloría de Cundinamarca (2016). Informe anual del estado de los recursos naturales y del ambiente del departamento de Cundinamarca. Vigencia 2015. Recuperado de http://contraloriadecundinamarca.gov.co/attachment/002\%20informes/008\%20 informe_a nual_del_estado_de_los_recursos_naturales_y_del_ambiente_del_ departamento_de_cund inamarca/2016/consolidado-provincias.html

Corporación Autónoma Regional de Cundinamarca (CAR). (2007). Diagnóstico, prospectiva y formulación de la cuenca hidrográfica del río Sumapaz. Bogotá: CAR.

Corporación Autónoma Regional de Cundinamarca (CAR). (2012). Plan de Gestión Ambiental Regional 2012-2023. Bogotá: CAR.

Crocker, J. y Luhtanen, R. (1990). Collective self-esteem and ingroup bias. Journal of personality and social psychology, 58(1), 60-67.

Edwards, M., Gil, D., Vilches, A. y Praia, J. (2004). La atención a la situación del mundo en la educación científica. Enseñanza de las Ciencias, 22(1), 47-64.

Euwater, R. (2005) Declaración europea por una nueva cultura del agua. Cuadernos del Cendes, 22(59), 161-163. Recuperado de http://www.redalyc.org/articulo. oa?id $=40305911>$ ISSN 1012-2508.

Folke, C. (2006). Resilience: The emergence of a perspective for social-ecological systems analyses. Global Environmental Change, 16, 253-267.

García Ballesteros, A. (2000). La cuestión ambiental en la geografía del siglo XX. Anales de Geografía de la Universidad Complutense, 20, 101-114.

Gómez, I. (2014). La acción colectiva del agua en Colombia y el referendo como acercamiento de democracia directa. Análisis Político, 27(80), 79-103.

Leff, E. (2004). Racionalidad ambiental y diálogo de saberes. Significancia y sentido en la construcción de un futuro sustentable. Polis Revista Académica Universidad Bolivariana de Chile, 2(7), 0.

Leff, E. (2006). Ética por la vida. Elogio de la voluntad de poder. Polis Revista Académica Universidad Bolivariana de Chile, 5(13), 0.

León, P. y Villarraga, F. (2014). El río Sumapaz. Proyecto hidroeléctrico "El Paso": Agua y vida campesina en riesgo. Bogotá: ILSA.

Maguire, B., y Cartwright, B. (2008). Assessing a community's capacity to manage change: a resilience approach to social assessment. Canberra: BRS Publication Sales.

Martínez-Alier, J. (2004). Los conflictos ecológico-distributivos y los indicadores de sustentabilidad. Revista Iberoamericana de Economía Ecológica, 1, 21-3. 
Marulanda, E. (1991). Colonización y conflicto: las lecciones del Sumapaz. Bogotá, Colombia: Tercer Mundo.

Molano, O. (2007). Identidad cultural un concepto que evoluciona. Opera, 7(7), 69-84.

Organización de las Naciones Unidas (ONU). (2010). Resolución /64/292. El derecho humano al agua y al saneamiento. Recuperado de http://www.un.org/ga/search/ view_doc.asp?symbol=A/RES/64/292\&Lang $=S$

Organización de las Naciones Unidas para la Educación, la Ciencia y la Cultura (Unesco). (2012). Managing Water under Uncertainty and Risk. United Nations World Water Development Report 4, vol. 1. París: World Water Assessment Program, United Nations Educational, Scientific and Cultural Organization.

Rapaport, C., Hornik-Lurie, T., Cohen, O., Lahad, M., Leykin, D. y Aharonson-Daniel, L. (2018). The relationship between community type and community resilience. International Journal of Disaster Risk Reduction, 31, 470-477.

Ribeiro, S. (2006). Las caras de la privatización del agua. En R. Grosse, C. Santos, J. Taks, y S. Thimmel (Coord.), Las Canillas Abiertas de América Latina II. La lucha contra la privatización del agua y los desafíos, (pp. 37-39). Montevideo, Uruguay: Casa Bertolt Brecht.

Roa, T. (2014). Petróleo. El nuevo mapa de los conflictos. En T. Roa y M. Navas (coords.), Extractivismo, conflictos y resistencias (pp. 199-238). Bogotá, Colombia: Censat Agua Viva - Amigos de la Tierra Colombia.

Roa García, M., Roa Avendaño, T. y Acosta, A. (2017). La democratización ambiental, pieza clave en el post-conflicto colombiano. En H. Alimonda, C. Toro y F. Martín (eds.), Ecología política latinoamericana: pensamiento crítico, diferencia latinoamericana y rearticulación epistémica ( $1^{\text {a }}$ ed., vol. 1, pp. 319-349). Buenos Aires: Clacso.

Romero, L. (19 de septiembre de 2014). Lucha por proteger el Sumapaz. El Espectador. Recuperado de https://www.elespectador.com/noticias/bogota/lucha-proteger-elsumapaz-articulo- 517608

Reich, J. W., Zautra, A. J. y Hall, J. S. (Eds.). (2010). Handbook of adult resilience. New York, EE. UU.: Guilford Press.

Santos, M. (1995). A questão do meio ambiente: desafios para a construção de una perspectiva transdisciplinar. Anales de Geografía de la Universidad Complutense, $1(1), 695-705$.

Sabatini, F. y Sepúlveda, C. (1997). Conflictos ambientales: entre la globalización y la sociedad civil. Santiago de Chile: Publicaciones CIPMA.

Shiva, V. (2003). Las guerras del agua. Privatización, contaminación y lucro. México D. F.: Siglo XXI.

Soja, E. W. y Hadjimichalis, C. (1979). Between geographical materialism and spatial fetishism. Antipode, 11(3), 59-67.

Suárez, N. (2001). Una concepción latinoamericana: la resiliencia comunitaria. En A. Melillo (comp.), Resiliencia. Descubriendo las propias fortalezas (3 ${ }^{\mathrm{a}}$ ed., pp. 72-81). Buenos Aires: Paidós. 
Suazo, M. (2016). Resiliencia comunitaria y su vinculación al contexto latinoamericano actual. Cuadernos de Trabajo Social, 14, 23-45.

Úcar, X. (2009). La comunidad como elecciyn: teorнa y prбctica de la acciyn comunitaria. Barcelona: Universitat Autònoma de Barcelona, Departament de Pedagogia Sistemàtica i Social. Recuperado de https://ddd.uab.cat/pub/recdoc/2009/54267/ comele_a2009.pdf

Uriarte, J. D. (2013). La perspectiva comunitaria de la resiliencia. Psicología Política, 47,7-18.

Vargas, R. (2006). La cultura del agua. Lecciones de la América indígena. Serie Agua y Cultura. Montevideo: Unesco - PHI.

Varela, L. y Duque, D. (2011). Estrategia de los agrarios de Sumapaz y Oriente del Tolima durante el Frente Nacional. Revista Historia y Sociedad, 21, 173-195.

Vicepresidencia de la República de Colombia, Observatorio del Programa Presidencial de Derechos Humanos y Derecho Internacional Humanitario (2002). Panorama actual de la región del Sumapaz. Bogotá: Fondo de Inversión para la Paz. 